\title{
Streptococcus mutans counts in plaque adjacent to orthodontic brackets bonded with resin-modified glass ionomer cement or resin-based composite
}

Solange Machado Mota ${ }^{(a)}$

Carla Enoki ${ }^{(b)}$

Izabel Yoko Ito (c)

Ana Maria Elias(d)

Mírian Aiko Nakane Matsumoto(e)

(a) Orthodontics Specialist; (b) MSc, Professor of Orthodontics, Department of Pediatric Dentistry; (e) PhD, Professor of Orthodontics, Department of Pediatric Dentistry - School of Dentistry of Ribeirão Preto, University of São Paulo.

(c) PhD, Professor of Microbiology, Department of Clinical Analysis, Toxicology and Bromatology, School of Pharmacy of Ribeirão Preto, University of São Paulo.

(d) PhD, Professor of Statistics, College of Science and Letters of Araraquara, University of São Paulo.

\author{
Corresponding author: \\ Mírian Aiko Nakane Matsumoto \\ Departamento de Clínica Infantil, \\ Odontologia Preventiva e Social \\ Faculdade de Odontologia de \\ Ribeirão Preto - USP \\ Avenida do Café, s/n \\ Ribeirão Preto - SP - Brazil \\ CEP: 14040-904 \\ E-mail: manakane@forp.usp.br
}

\begin{abstract}
This study investigated the number of Streptococcus mutans CFU (colony forming units) in the saliva and plaque adjacent to orthodontic brackets bonded with a glass ionomer cement - GIC (Fuji Ortho) or a resin-based composite - RC (Concise). Twenty male and female patients, aged 12 to 20 years, participated in the study. Saliva was collected before and after placement of appliances. Plaque was collected from areas adjacent to brackets and saliva was again collected on the $15^{\text {th }}, 30^{\text {th }}$, and $45^{\text {th }}$ day after placement. On the $30^{\text {th }}$ day, $0.4 \%$ stannous fluoride gel was applied for 4 minutes. No significant modification in the number of Streptococcus mutans CFU in saliva was observed after placement of the fixed orthodontic appliances. On the $15^{\text {th }}$ day, the percentage of Streptococcus mutans CFU in plaque was statistically lower in sites adjacent to GIC-bonded brackets (mean $=0.365$ ) than in those adjacent to RCbonded brackets (mean $=0.935$ ). No evidence was found of a contribution of GIC to the reduction of CFU in plaque after the $15^{\text {th }}$ day. Topical application of stannous fluoride gel on the $30^{\text {th }}$ day reduced the number of CFU in saliva, but not in plaque. This study suggests that the antimicrobial activity of GIC occurs only in the initial phase and is not responsible for a long-term anticariogenic property.
\end{abstract}

Descriptors: Streptococcus mutans; Orthodontic appliances; Glass ionomer cements; Tin fluorides. 


\section{Introduction}

Enamel decalcification during orthodontic treatment is a serious clinical problem, in particular in patients with poor oral hygiene habits. Recent studies have reported that demineralization of dental surfaces during treatment can be found in 50 to $75 \%$ of all patients with fixed orthodontic appliances. ${ }^{1-3}$

Clinically, white-spot lesions can be seen around brackets. These lesions are incipient carious lesions that can be remineralized by application of fluoride. ${ }^{4,5}$ Fluoride-releasing bonding materials and cements have been used because they reduce the need for patient compliance and potentially inhibit demineralization. ${ }^{1-3,6-9}$

The reduction in decalcification observed when brackets are bonded with fluoride-containing materials results from the slow and constant release of fluoride, even if in small amounts, in the exact sites where there is higher cariogenic risk. Such reduction may also result from the changes caused on enamel surfaces by the initial higher concentrations of fluoride. ${ }^{4,10,11}$

Fluoride-releasing bonding materials also stimulate the development of a calcium fluoride layer on enamel surfaces adjacent to brackets. This layer serves as a potential reserve of fluoride, which slowly releases fluoride ions during the demineralization and remineralization processes. It also acts as a barrier against acid challenge. ${ }^{4,12,13}$

The oral environment of orthodontic patients undergoes changes, such as $\mathrm{pH}$ reduction, larger number of sites available for Streptococcus mutans collection, and increased accumulation of food particles, which may lead to an increased number of Streptococcus mutans colony-forming units (CFU) in saliva ${ }^{14,15}$ Such changes may contribute to the development of the decalcification lesions frequently found at the end of orthodontic treatments. ${ }^{14,16}$

Several studies have confirmed increases in the number of Streptococcus mutans in saliva during orthodontic treatment. However, these studies have not investigated the use of glass-ionomer cements (GIC) in the placement of orthodontic appliances. ${ }^{14,17}$

Furthermore, scarce data about the use of GIC in bracket bonding have been made available so far. Studies in the literature report that fluoride released from restorations with conventional GIC ${ }^{18-21}$ and brackets bonded with GIC T2-28 $^{22}$ ay have a local cariostatic effect when cariogenic challenge is increased during orthodontic treatment. Therefore, white spots, which are signs of decalcification, may be prevented.

The purpose of this study was to investigate whether fluoride released from GIC resulted in lower Streptococcus mutans counts in plaque adjacent to GIC-bonded brackets than in that adjacent to brackets bonded with resin composites. This study also investigated the effect of a topical application of $0.4 \%$ stannous fluoride gel.

\section{Material and Methods}

Following the approval of the research project by the Ethics Committee of the School of Dentistry of Ribeirão Preto, University of São Paulo (process \# 2001.1.827.58.7), 20 male and female patients, aged 12 to 20 years, were selected from the group of patients seen in the Orthodontics Clinic of that institution.

To determine the number of Streptococcus mutans CFU, $2.0 \mathrm{ml}$ of non-stimulated saliva were collected from each patient before placement of the appliance. The collected material was placed in properly labeled $15 \times 100 \mathrm{~mm}$ sterile tubes containing 4 to 5 glass beads. The samples were processed at the Microbiology Laboratory of the School of Pharmacy of Ribeirão Preto, University of São Paulo.

Placement of appliances was performed, and brackets were bonded with a resin-based composite (Concise, 3M-Unitek, Sumaré, São Paulo, Brazil) in one side of the dental arch, and with a GIC (GC Fuji Ortho LC, GC Corporation, Tokyo, Japan) in the other side. The bonding material used in each quadrant was: upper left $=$ Fuji Ortho; upper right $=$ Concise; lower left $=$ Concise; and lower right $=$ Fuji Ortho.

After the placement of appliances was completed, saliva was collected, and oral hygiene instructions were given to the patient. On the $15^{\text {th }}$ and $30^{\text {th }}$ days after placement, saliva was collected again, and plaque was removed from the areas adjacent to the brackets on the right maxillary canine, left maxil- 
lary lateral incisor, left mandibular central incisor, and right mandibular lateral incisor. Collection was performed continuously with a sterilized probe.

On the $30^{\text {th }}$ day, $0.4 \%$ stannous fluoride gel was topically applied for 4 minutes. Saliva and plaque were collected for the last time on the $45^{\text {th }}$ day.

The plaque removed was spread on $15 \times 100 \mathrm{~mm}$ sterile test tubes containing 4 to 5 glass beads and $2.0 \mathrm{ml}$ of phosphate buffered saline solution (PBS). The test tubes were sent to the laboratory for microbiological processing.

Saliva and plaque samples were vortexed for two and one minutes, respectively, for dispersion, and submitted to tenfold serial dilutions. After that, $50 \mathrm{Pl}$ of each dilution was plated equidistantly on SB20 agar (tryptone soy yeast agar plus $20 \%$ sucrose and $0.2 \mathrm{U} / \mathrm{mL}$ Bacitracin; Sigma - St. Louis, $\mathrm{MO}$ ) and incubated under candle jar system at $37 \mathrm{qC}$ for 2 to 3 days.

\section{Statistical analysis}

For statistical analysis, the original data, recorded as CFU (colony-forming units), were transformed into $\log _{10}(\mathrm{CFU})$, and all results were expressed as $\log (\mathrm{CFU})$. To analyze statistically the materials' effect on CFU counts in the biofilm, teeth with brackets retained with the same material in a same patient were considered as repetitions of measurements. Therefore, $\log (\mathrm{CFU})$ was calculated considering the means of CFU counts for brackets bonded with the same material.

The hypothesis of equality of $\log (\mathrm{CFU})$ means in saliva in the beginning of the treatment and at the three moments of biofilm collection (i.e., 15, 30 and 45 days after installation of the orthodontic appliances) was tested using the Hotelling's T2 multivariate test for comparison of means of repeated measurements. To test the hypothesis that the resinmodified GIC presents greater protection against Streptococcus mutans in the biofilm only in the beginning of the treatment, $\log (\mathrm{CFU})$ means in the biofilm adjacent to both materials were compared at each moment, separately. Paired Student's $t$-test was used to test the hypothesis of equality between the materials regarding $\log (\mathrm{CFU})$ means in the biofilm, at each moment of collection.

\section{Results}

Streptococcus mutans CFU counts in saliva (in the beginning of the treatment and 15, 30 and 45 days after installation of the orthodontic appliances) showed that all patients presented moderate to high caries risk throughout the evaluation period (Table 1). Hotelling's T2 multivariate test did not show statistically significant differences among the $\log (\mathrm{CFU})$ means in saliva at any of the collection moments $(\mathrm{F}=2.298 ; \mathrm{gl}=3$ and $15 ; \mathrm{p}=0.119)$, which means that there was no evidence of remarkable alterations in the patients' saliva with respect to the CFU counts throughout the study.

Regarding the effect of the tested materials on CFU formation, it was observed that $\log (\mathrm{CFU})$ means in the biofilm adjacent to Fuji Ortho LC 15 days after the beginning of the treatment was significantly lower than the $\log (\mathrm{CFU})$ means in the biofilm adjacent to Concise $\left(\mathrm{t}=2.23^{\mathrm{a}} ; \mathrm{df}=17 ; \mathrm{p}=0.039\right.$ ) (Table 2). The results also revealed that there were no statistically significant differences among the $\log (\mathrm{CFU})$ means in the biofilm of brackets retained with Concise and Fuji Ortho LC, at 30 and 45 days after placement of the appliances. $\log (\mathrm{CFU})$ and the respective means in the biofilm of teeth bonded with Concise and Fuji Ortho LC at the three moments of collection are given in Graph 1.

\section{Discussion}

The most important reason to use fluoride-containing materials is their anticariogenic activity. Such activity can be monitored by means of longitudinal studies about fluoride release, fluoride effects on demineralization, the quality and amount

Table 1 - Means ( \pm SD), minimum and maximum Streptococcus mutans $\log _{10}$ (CFU) values in saliva, at the beginning of treatment and 15, 30 and 45 days after placement of the appliances.

\begin{tabular}{l|c|c|c}
\hline Time of treatment & Means $(\mathrm{r}$ SD) & Minimum & Maximum \\
\hline Beginning & $5.088 \mathrm{r} 0.884$ & 3.000 & 6.556 \\
\hline 15 days & $5.279 \mathrm{r} 1.105$ & 3.146 & 7.025 \\
\hline 30 days & $5.505 \mathrm{r} 0.789$ & 3.763 & 7.182 \\
\hline 45 days & $5.323 \mathrm{r} 0.931$ & 3.255 & 6.991 \\
\hline
\end{tabular}

Hotelling's T2 multivariate test: $F=2.298 ; g l=3$ and $15 ; p=0.119$. 


\begin{tabular}{|c|c|c|c|c|c|c|c|}
\hline \multirow{7}{*}{$\begin{array}{l}\text { Table } 2 \text { - Descriptive statistics } \\
\text { of } \log _{10} \text { (CFU) values in biofilm } \\
\text { for type of material and time of } \\
\text { treatment and paired Student's } \\
\text { t-test for comparison of means } \\
15,30 \text { and } 45 \text { days after } \\
\text { placement of the appliances. }\end{array}$} & Time & Material & Means (r SD) & Minimum & Maximum & $t(d f=17)$ & $\mathrm{p}$ \\
\hline & \multirow{2}{*}{15 days } & GIC & 3.366 r 0.390 & 2.540 & 4.020 & \multirow{2}{*}{2.231} & \multirow{2}{*}{$0.039 *$} \\
\hline & & Concise & 3.747 r 0.684 & 2.651 & 4.917 & & \\
\hline & \multirow{2}{*}{30 days } & GIC & 3.645 r 0.460 & 2.858 & 4.512 & \multirow{2}{*}{0.605} & \multirow{2}{*}{0.553} \\
\hline & & Concise & 3.724 r 0.552 & 2.801 & 4.785 & & \\
\hline & \multirow{2}{*}{45 days } & GIC & 3.666 r 0.488 & 3.047 & 4.563 & \multirow{2}{*}{0.479} & \multirow{2}{*}{0.638} \\
\hline & & Concise & 3.747 r 0.654 & 2.69 & 4.880 & & \\
\hline
\end{tabular}

*Significance level: $p<0.05$

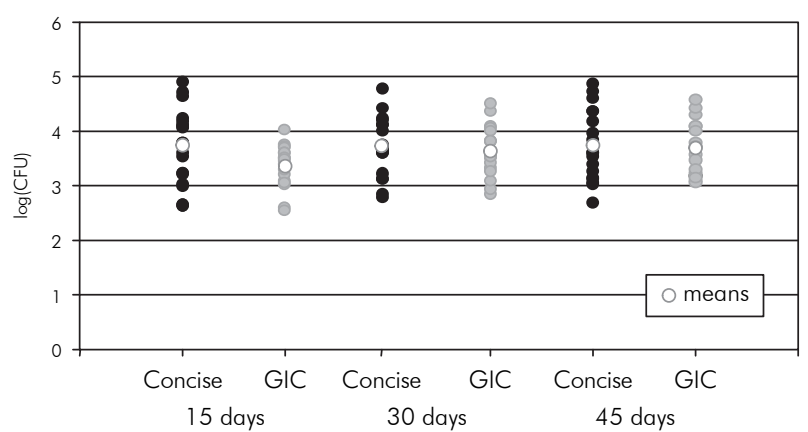

Graph 1 - Log(CFU) values according to material and time of treatment and respective means.

of plaque, or, finally, the occurrence of secondary caries adjacent to bonding material.

Streptococcus mutans is also found on healthy surfaces, and its presence does not always indicate the presence of active caries. However, an increased number of these microorganisms on any surface indicates that disease may be present or may develop in the near future. ${ }^{14}$

Clinical evaluations of GIC have yielded controversial results. Some evidence points towards the reduction of the risk of caries on tooth surfaces adjacent to glass ionomer restorations. ${ }^{18,19,21,27}$

After orthodontic treatment and before appliances were removed (mean: 9.5 months), the percentage of total Streptococcus mutans CFU found in bacterial plaque was lower in areas adjacent to brackets bonded with GIC than in those adjacent to surfaces bonded with a resin composite. ${ }^{27}$

In our clinical study, statistically significant differences between the two types of material used to bond brackets were found only on the $15^{\text {th }}$ day after placement of the appliance, when there was a signif- icant reduction in the number of $\mathrm{CFU}$ in the plaque collected from areas adjacent to brackets bonded with resin-modified GIC. No significant difference was found at the other times. Matalon et al. ${ }^{26}$ (2005) related that reinforced GIC (Fuji Ortho LC) exhibited potent antibacterial activity, which lasted 1 week and diminished over the next 3 weeks.

Fluoride release from GIC was directly associated with its antimicrobial activity, that is, when $\mathrm{pH}$ is close to neutral $(7.1-7.3)$ and the amount of fluoride is $140 \mathrm{r} 25 \mathrm{ppm}$. Therefore, GIC may be effective for a short period of time, maybe for only a few days, as shown in our study. ${ }^{28}$

Fishman, Tinanoff ${ }^{23}$ (1994) found no association between the amount of fluoride released and antimicrobial activity of resin-modified GIC in vitro. On the contrary, the bacterial growth inhibiting effect seemed to be associated with GIC acid release. The reduction in resin-modified GIC $\mathrm{pH}$ and the size of bacterial growth inhibition areas are consistently associated. The largest amount of acid release from resin-modified GIC and its greatest antimicrobial activity are found immediately after the material is used. As time passes, less acid is released and bacterial growth inhibition decreases. A reduced inhibiting effect for Streptococcus mutans seems to be associated with the fact that these microorganisms are acid-tolerant.

After application of fluoride on old GIC fillings, there was no significant increase in fluoride concentration on bacterial plaque. ${ }^{24}$ Our results are in agreement with the findings of the study cited above - the topical application of stannous fluoride gel in our study did not significantly reduce the number of Streptococcus mutans CFU in plaque. In contrast, 
there were statistically significant differences in the number of Streptococcus mutans CFU in the saliva of patients after fluoride application. However, Corry et al. ${ }^{22}$ (2003) concluded that fluoride release from Fuji Ortho LC alone fell to minimal values, but with daily addition of extrinsic fluoride the levels fell initially and then followed an upward trend.

Growth of Streptococcus mutans in plaque was inhibited in vivo in areas adjacent to restorations with conventional GIC and with glass-ionomer silver cement. ${ }^{19,20,29}$ However, Van Dijken et al. ${ }^{15}$ (1991) did not find any reduction in Streptococcus mutans or Lactobacilli in plaque collected from 1year-old GIC restorations.

Our study found a smaller number of Streptococcus mutans CFU in plaque adjacent to brackets bonded with resin-modified GIC than in plaque adjacent to resin composite only on the $15^{\text {th }}$ day after placement of the appliance. The resin-modified GIC antimicrobial activity fell along time, and was not reestablished when $0.4 \%$ stannous fluoride gel was topically applied on the $30^{\text {th }}$ day.

The antimicrobial activity of resin-modified GIC was only observed in the initial phase of the study, for a short period of time; it does not, thus, seem to be responsible for resin-modified GIC's anticariogenic activity in the long run. Gorton, Featherstone $e^{10}$ (2003) found, in an in vivo study, that the resinmodified GIC's cariostatic effect around brackets could be observed up to 4 weeks after placement. Maybe resin-modified GIC activity in the process of caries development is associated with its slow fluoride release, which results in the presence of fluoride in enamel or in plaque fluid during the soluble phase. Therefore, it may inhibit demineralization

\section{References}

1. Ögaard B, Arends J, Helseth H, Dijkman G, Van Der Kuijl M. Fluoride level in saliva after bonding orthodontic brackets with a fluoride containing adhesive. Am J Orthod Dentofacial Orthop. 1997;111(2):199-202

2. Ögaard B, Rölla G, Arends J. Orthodontic appliances and enamel demineralization. Part 1. Lesion development. Am J Orthod. 1988;94(1):68-73.

3. Ögaard B, Rölla G, Arends J, Ten Cate JM. Orthodontic appliances and enamel demineralization. Part 2. Prevention and promote remineralization in sites of highest cariogenic risk. ${ }^{3}$

Fluoride activity in remineralization is a result of fluoride's behavior as a catalyst, which is capable of lowering the activation energy required for crystal growth. ${ }^{5}$ The size and electrostatic charge of fluoride permit a more favorable three-dimensional arrangement of calcium and phosphate on the crystal surface.

Further in vivo studies about the long-term effect of resin-modified GIC on plaque should attempt to define resin-modified GIC's actual antimicrobial effect, as well as its magnitude, and how it affects bacterial cells. Although several synergistic and antagonistic factors affect clinical studies about the effect of a material on bacterial growth, only clinical studies are able to provide an explanation for the cariostatic potential of a material.

\section{Conclusion}

After placement of fixed orthodontic appliances, a significant modification in the number of Streptococcus mutans CFU in saliva was not observed. The number of Streptococcus mutans CFU in plaque adjacent to brackets bonded with resin-modified GIC was smaller than in plaque adjacent to brackets bonded with resin-based composite only on the $15^{\text {th }}$ day after placement of the appliance. Topical application of $0.4 \%$ stannous fluoride gel on the $30^{\text {th }}$ day did not affect the number of Streptococcus mutans in plaque; the number of microorganisms in saliva, however, was reduced. This study suggests that the antimicrobial activity of resin-modified GIC occurs only on the initial phase and is not responsible for a long-term cariostatic potential.

and treatment of lesions. Am J Orthod Dentofacial Orthop. 1988;94(2):123-8.

4. Basdra EK, Huber H, Komposch G. Fluoride released from orthodontic bonding agents alters the enamel surface and inhibits enamel demineralization in vitro. Am J Orthod Dentofacial Orthop. 1996;109(5):466-72.

5. Proctor \& Gamble Inc. In: Kofman SH, Suljack JP, Koch G. Remineralization of natural carious lesions with a glass ionomer cement. Swed Dent J. 1991;99(1):13-9. 
6. Geiger AM, Gorelick L, Gwinnet AJ, Griswold PG. The effect of a fluoride program on white spot formation during orthodontic treatment. Am J Orthod. 1988;93(1):29-37.

7. Papagiannoulis L, Kakaboura A, Eliades G. In vivo vs in vitro anticariogenic behavior of glass-ionomer and resin composite restorative materials. Dent Mater. 2002;18(8):561-9.

8. Wiltshire WA, Janse Van Rensburg S. Fluoride release from visible light-cured orthodontic adhesive resins. Am J Orthod Dentofacial Orthop. 1995;108(3):278-83.

9. Zachrisson BU. Fluoride application procedures in orthodontic practice: current concepts. Angle Orthod. 1975;45(1):7281.

10. Gorton J, Featherstone JD. In vivo inhibition of demineralization around orthodontic brackets. Am J Orthod Dentofacial Orthop. 2003;123(1):10-4.

11. Pascotto RC, Navarro ME, Capelozza Filho L, Cury JA. In vivo effect of a resin-modified glass ionomer cement on enamel demineralization around orthodontic brackets. Am J Orthod Dentofacial Orthop. 2004;125(1):36-41.

12. Boeckh C, Schumacher E, Podbielski A, Haller B. Antibacterial activity of restorative dental biomaterials in vitro. Caries Res. 2002;36(2):101-7.

13. Rix D, Foley TF, Banting D, Mamandras A. A comparison of fluoride release by resin-modified GIC and polyacidmodified composite resin. Am J Orthod Dentofacial Orthop. 2001;120(4):398-405.

14. Rosenbloom RG, Tinanoff N. Salivary Streptococcus mutans levels in patients before, during, and after orthodontic treatment. Am J Orthod Dentofacial Orthop. 1991;100(1):35-7.

15. Van Dijken J, Persson S, Sjöström S. Presence of Streptococcus mutans and lactobacilli in saliva and on enamel, glass ionomer cement, and composite resin surfaces. Scand J Dent Res. 1991;99(1):13-9.

16. Stratemann MW, Shannon IL. Control of decalcification in orthodontic patients by daily self-administered application of a water-free 0.4 per cent stannous fluoride gel. Am J Orthod. 1974;66(3):273-9.

17. Vierrou AM, Manwell MA, Zamek RL, Sachdeva R, Tinanoff N. Control of Streptococcus mutans with topical fluorides in patients undergoing orthodontic treatment. J Am Dent Assoc. 1986;113(4):644-6.
18. Qvist V, Laurberg L, Pousen A, Teglers PT. Longevity and cariostatic effects of everyday conventional glass ionomer and amalgam restorations in primary teeth: three-year results. J Dent Res. 1997;76(7):1387-96.

19. Svanberg M. Class II amalgam restorations, glass ionomer tunnel restorations, and caries development on adjacent tooth surfaces: a three-year clinical study. Caries Res. 1992;26(4):3158.

20. Svanberg M, Krasse B, Örnerfeldt HO. Mutans streptococci in interproximal plaque from amalgam and glass ionomer restorations. Caries Res. 1990;24(2):133-6.

21. Tyas MJ. Cariostatic effect of glass ionomer cement: a five-year clinical study. Aust Dent J. 1991;36(3):236-9.

22. Corry A, Millett DT, Creanor SL, Foye RH, Gilmour WH. Effect of fluoride exposure on cariostatic potential of orthodontic bonding agents: an in vitro evaluation. J Orthod. 2003;30(4):323-9.

23. Fishman SA, Tinanoff N. The effect of acid and fluoride release on the antimicrobial properties of four glass ionomer cements. Pediatr Dent. 1994;16(5):368-70.

24. Forss H, Jokinen J, Spets-Happonen S, Seppä L, Luoma H. Fluoride and mutans streptococci in plaque grown on glass ionomer and composite. Caries Res. 1991;25(6):454-8.

25. Friedl KH, Schmalz G, Hiller KA, Shams M. Resin-modified glass ionomer cements: fluoride release and influence on Streptococcus mutans growth. Eur J Oral Sci. 1997;105(1):81-5.

26. Matalon S, Slutzky H, Weiss EI. Antibacterial properties of 4 orthodontic cements. Am J Orthod Dentofacial Orthop. 2005;127(1):56-63.

27. Örtendahl T, Thilander B, Svanberg M. Mutans Streptococci and incipient caries adjacent to glass ionomer cement or resinbased composite in orthodontics. Am J Orthod Dentofacial Orthop. 1997;112(3):271-4.

28. Rodriguez JPL, Godoy FG, Lindquist R. Growth inhibition of glass ionomer cements on mutans streptococci. Pediatr Dent. 1994;16(5):346-9.

29. Forss H, Näse L, Seppä L. Fluoride concentration, mutans streptococci and lactobacilli in plaque from old glass ionomer fillings. Caries Res. 1995;29(1):50-3. 